\title{
Nivel de influencia de los medios de comunicación sobre la alimentación saludable en adolescentes de colegios públicos de un distrito de Lima
}

\author{
Media influence on adolescents' healthy nutrition in Lima district public schools
}

\author{
Verónica Román¹, Margot Quintana ${ }^{1,2}$ \\ ${ }^{1}$ Escuela Académico Profesional de Nutrición, Facultad de Medicina, UNMSM. Lima, Perú. \\ ${ }^{2}$ Departamento de Nutrición, Facultad de Medicina, UNMSM. Lima, Perú.
}

\begin{abstract}
Resumen
Introducción: Los medios de comunicación tienen gran influencia en el comportamiento de adolescentes; sus mensajes pueden ser potenciadores 0 perjudiciales para su salud. Objetivos: Determinar el grado de influencia que ejercen los medios de comunicación tanto en estudiantes pertenecientes a escuelas promotoras para el desarrollo sostenible (EDPS) como en aquellos que no pertenecen, así como cuáles son aquellos que más influyen sobre alimentación saludable en estudiantes de nivel secundario. Además, determinar si la condición de EDPS se encuentra asociada a una mayor o menor influencia de los medios de comunicación. Diseño: Estudio observacional, descriptivo y transversal. Lugar: Instituciones Educativas del distrito Puente Piedra. Participantes: Adolescentes de ambos sexos. Intervenciones: Trescientos treinta adolescentes fueron elegidos por conveniencia y distribuidos equitativamente entre pertenecientes y no pertenecientes a EPDS. Cada adolescente respondió un cuestionario sobre la influencia percibida de medios de comunicación acerca de alimentación saludable. Se aplicó chi cuadrado para asociar niveles y Man-Whitney, para comparar puntajes. Principales medidas de resultados: Nivel de influencia percibida de medios de comunicación, cuya puntuación fue: 0 a 8 (bajo), 9 a 16 (medio), 17 a 24 (alto). Resultados: El nivel de influencia 'medio' obtuvo el mayor porcentaje en ambos grupos. El promedio de puntaje fue 10,9 para los pertenecientes y 10,6 para los no pertenecientes a EPDS, sobre un máximo de 24 puntos. El medio de comunicación que más influyó en la elección de los alimentos para ambos grupos fue la televisión. El que menos influyó para los pertenecientes fue el diario y para los no pertenecientes, la radio. La familia influenciaría más en la elección de alimentos en los pertenecientes a EPDS y los medios de comunicación en los no pertenecientes. Conclusiones: El nivel de influencia de los medios de comunicación sobre la alimentación saludable fue de nivel 'medio' para ambos grupos de adolescentes de nivel secundario. El medio de comunicación que más influyó en la elección de los alimentos para ambos grupos fue la televisión. Para ambos grupos, el entorno familiar tiene un rol educador sobre el consumo de alimentos saludables.

Palabras clave: Medios de comunicación; conducta del adolescente; alimentos
\end{abstract}

\begin{abstract}
saludables.
Abstract

Introduction: Media have influence on the behavior of adolescents, and their messages can be enhancers or harmful to their health. Objectives: To determine the association of perceived media influence on healthy eating and belonging to Promoting Schools for Sustainable Development in adolescents. Desing: Descriptive, transversal and cross-association study. Location: Educational Institutions in Puente Piedra district. Participants: Male and female adolescents. Interventions: Three hundred and thirty adolescents chosen for convenience were divided equally whether they belonged or not to the program. Each adolescent answered a questionnaire about how they perceived media influence on healthy nutrition. Chi square was used to associate levels and Man-Whitney to compare scores between both groups. We used Excel 2003 and SPSS software 13.0. Main outcome measures: Scores for perceived media influence were 0-8 (low), 9-16 (medium), and 17-24 (high). Results: The 'medium' level had the highest percentage in both groups. The average score was 10.9 for those belonging to the program and 10.6 for those not belonging. The media that most influenced in the choice of food for both groups was television. The media that less influenced for those belonging to the program was newspaper and for those not belonging the radio. The family would influence in the choice of food in those belonging to the program and the media in those not belonging. Conclusions: There was no statistically significant difference between both groups.
\end{abstract}

Key words: Communications media; adolescent behavior; health food.

\section{INTRODUCCIÓN}

Las instituciones educativas (IE) son escenarios donde se aplica la promoción de la salud. Allí se desarrollan acciones de aprendizaje creadas para ampliar el conocimiento, comprensión y habilidades personales del adolescente, a manera de facilitar cambios de conducta que promuevan estilos de vida adecuados, como por ejemplo una alimentación saludable ${ }^{(1)}$.
En el año 2006, se integraron los programas "Escuelas promotoras de salud" y "Escuelas saludables". La primera es ejecutada por el Hospital Puente Piedra, en la Red del mismo nombre -que comprende los distritos de Puente Piedra, Santa Rosa, Ancón y parte de Carabayllo-, y la segunda es ejecutada por la Unidad de Gestión Educativa Local (UGEL) $\mathrm{N}^{\circ} 4$, cuya jurisdicción abarca también los distritos antes mencionados. Esta unión de programas posteriormente se denominaría "Escuelas promotoras para el desarrollo sostenible" (EPDS) (2) y cuenta con la participación y auspicio de la Organización Humanitaria Internacional Plan Perú y la Dirección de Salud (DISA) V Lima Ciudad. Para el año 2009, existían 50 IE de nivel inicial, primario y secundario, dentro del programa EPDS. De ellas, 45 fueron clasificadas dentro de 3 niveles (I, II y III) previas a ser declaradas propiamente EPDS y 5 ya declaradas como tales. De las 28 IE de 
nivel secundario del distrito de Puente Piedra, 18 estaban dentro del programa. En estas últimas se trata el tema de alimentación y nutrición.

Entendemos por alimentación saludable aquella que es variada, aporta energía y todos los nutrientes esenciales que cada persona necesita para mantenerse sana, permitiéndole una mejor calidad de vida en todas las edades; ello previene enfermedades, como desnutrición, obesidad, hipertensión y algunos tipos de cáncer ${ }^{(3)}$.

En lo que se refiere a la alimentación del adolescente, el Ministerio de Salud (MINSA) ${ }^{(4)}$ recomienda comer la mayor variedad posible de alimentos, guardar un equilibrio entre lo que se come y la actividad física, elegir una dieta abundante en cereales, verduras y frutas, pobre en grasa saturada, sal, y que proporcione suficiente calcio y hierro para satisfacer los requerimientos de un organismo en crecimiento. Los refrigerios escolares también deben proporcionar una nutrición completa con alimentos en su forma natural y/o en algunas preparaciones industriales, que según el Centro Nacional de Alimentación y Nutrición (CENAN) pueden ser yogurt envase individual (sachet o vasito), jugos en sachet o tetrapack con contenido bajo de azúcar y leche envasada UHT, tipo sachet ${ }^{(5)}$.

Actualmente, el tema de alimentación saludable es difundido por diferentes medios de comunicación de tipo masivo, como impresos (diarios o periódicos, revistas, entre otros), radio, televisión e internet ${ }^{(6)}$. Los medios de comunicación tienen gran influencia en las orientaciones hacia el consumo y en los comportamientos de la población juvenil, por lo que sus mensajes pueden ser potenciadores o perjudiciales para la salud $^{(7,8)}$.

$\mathrm{Al}$ identificar qué medios tienen más influencia en la elección de los alimentos en los estudiantes de nivel secundario, estos pueden ser utilizados en la difusión de temas de alimentación saludable y con ello contribuir con el propósito de disminuir problemas nutricionales, como el sobrepeso y la obesidad. En nuestro país, el sobrepeso y obesidad en adolescentes han incrementado en los últimos 30 años; así, en mujeres de 10 a 14 años se incrementó de 11,2 a 18,6\%, y en varones de 4 a $9,7 \%^{(9,10)}$. A nivel poblacional, Lima es uno de los departamentos con más alta prevalencia de sobrepeso, 17,24\%, a predominio urbano ${ }^{(11)}$

Un estudio cubano señala que los adolescentes adquieren conocimientos sobre el consumo de vegetales a través de la televisión (95\%), seguido de la escuela $(79 \%)$, la radio $(62 \%)$ y la familia $(52 \%)^{(12)}$; estos resultados coinciden con los de otros dos estudios realizados en México y Cuba, que muestran que los medios de comunicación masiva, en especial la televisión, tienen efectos importantes sobre el comportamiento y las creencias de las personas, constituyendo un medio potencial de excelencia por su grado de motivación y eficacia para promover el aprendizaje, que supera frecuentemente las posibilidades de la familia y la escuela ${ }^{(13,14)}$. Asimismo, un estudio español destaca que 35,2\% de alumnos no reconoce la influencia de la TV en sus hábitos alimentarios; sin embargo, $71,8 \%$ consume productos difundidos en anuncios de TV ${ }^{(15)}$.

Así, el objetivo de la presente investigación fue determinar el grado de influencia que ejercen los medios de comunicación tanto en estudiantes pertenecientes a EDPS como en aquellos que no pertenecen, así como cuáles son aquellos que más influyen sobre alimentación saludable en estudiantes de nivel secundario. Además, determinar si la condición de EDPS se encuentra asociada a una mayor o menor influencia de los medios de comunicación.

\section{MÉTODOS}

Se realizó un estudio observacional, descriptivo y transversal ${ }^{(16)}$. De la población de 4502 adolescentes que cursaban del $3^{\circ}$ al $5^{\circ}$ grado de nivel secundario de todas las IE públicas del distrito de Puente Piedra, se tomó una muestra de 330 adolescentes, mediante muestreo por conveniencia y cuotas. De ellos, la mitad (165) fue de 7 IE pertenecientes al programa EPDS (PP) y la otra mitad (165), de 7 IE no pertenecientes a dicho programa (NPP). Se empleó la fórmula de tamaño de muestra para estimar una proporción ${ }^{(16)}$. Se consideró un nivel de confianza del 95\%.

Se aplicó una encuesta durante los meses de junio a agosto del año 2009. Previamente se validó el instrumento mediante juicio de expertos y una prueba piloto ${ }^{(17)}$. En el juicio de expertos participaron docentes de la Escuela Acádemico Profesional de Nutrición de la Universidad Nacional Mayor de San Marcos y una comunicadora social. La prueba piloto se realizó en una población pequeña de estudiantes de uno de los colegios evaluados.

Con la autorización de los directores de cada IE y el consentimiento de los estudiantes a encuestar, se procedió a aplicar el cuestionario sobre la influencia percibida por parte de los medios de comunicación, la misma que estuvo conformada por 9 preguntas en total. Las dos primeras abordan la influencia que la televisión, radio, diarios e internet tendrían en la elección de sus alimentos, las dos siguientes sobre aquellos alimentos saludables y no saludables que los adolescentes manifestaron que cada medio de comunicación influenció en su mayor consumo. Dos preguntas se dirigieron a determinar aquellos programas de TV, radio, secciones de diarios o páginas web donde hubieran encontrado información sobre alimentos saludables y no saludables. Las siguientes dos preguntas fueron sobre el medio de comunicación que creían que más influye en la elección de alimentos, si compraron o desearon comprar algún alimento publicitado y cuáles fueron. La última pregunta fue si los medios de comunicación, familia, escuela o amistades influyen en ellos para consumir un determinado alimento. Cada estudiante era identificado mediante códigos.

Las respuestas a las primeras 4 preguntas fueron evaluadas con un puntaje de 0 cuando 'no influye', 2 cuando 'influye poco', 4 cuando 'influye regular' o 6 cuando 'influye mucho', por lo que el nivel de influencia percibida por los medios de comunicación fue categorizado como 'bajo' (0 a 8 puntos), 'medio' (9 a 16 puntos) y 'alto' (17 a 24 puntos). 
El resto de preguntas no tuvo puntaje, por lo que se contabilizó las respuestas según alternativas; por ejemplo, $70 \%$ de los adolescentes pertenecientes a EPDS indicaron que la televisión fue el medio de comunicación que más influía en la elección de sus alimentos. Tanto los puntajes de cada respuesta y los puntos de corte fueron determinados de forma arbitraria por parte de los investigadores del presente estudio, en base a la prueba piloto.

Para el análisis de datos, se utilizó el paquete estadístico SPSS 13.0. Se presenta los datos mediante estadística descriptiva: media, desviación estándar, porcentajes, tablas de frecuencia y gráficos de barras. Para asociar la influencia percibida por los medios de comunicación sobre alimentación saludable, según su pertenencia a EPDS, se empleó la prueba chi cuadrado. Se comparó los puntajes de la influencia percibida de los medios de comunicación utilizando la prueba Mann-Whitney, ya que la distribución de los datos no era normal. Para el análisis inferencial, se empleó un nivel de significancia $p<0,05$ para determinar la existencia de diferencias estadísticamente significativas.

\section{RESULTADOS}

Participaron del estudio 330 adolescentes estudiantes de nivel secundario (161 mujeres y 169 varones), cuyas edades se encontraron entre los 13 y 19 años. Para la presentación y análisis de datos hallados, se tomó en cuenta dos grupos; así, de los 330 adolescentes, la mitad (165) era de 7 IE pertenecientes al programa EPDS (PP) y la otra mitad (165), de 7 IE no pertenecientes a dicho programa (NPP).

El promedio de puntaje de la encuesta fue 10,9 para los PP y 10,6 para los NPP. Sobre los medios de comunicación y su influencia en la alimentación saludable, el nivel de influencia 'medio' fue el que obtuvo mayor porcentaje y el nivel de influencia 'alto' tuvo menor porcentaje en ambos grupos (figura 1).

$\mathrm{Al}$ desarrollar al detalle las preguntas de la encuesta aplicada observamos que, por ejemplo -según el medio de comunicación preguntado (tv, radio, diarios, internet)-, ambos grupos (PP y NPP) indicaron en mayor porcentaje (70\%) que la televisión tuvo influencia regular. En cuanto a la radio, el 33,3\% de adolescentes PP indicó que influye poco, a diferencia de los NPP que indicaron en $36,4 \%$ que no influye. En cuanto a los diarios, más de $50 \%$ de los adolescentes PP percibía que no influye o que la influencia era poca, mientras que el mayor porcentaje de adolescentes NPP indicó que la influencia era regular. El 28,5\% de los adolescentes PP y el 36,4\% de los NPP percibían que el internet no influye en la elección de sus alimentos.

Los adolescentes de ambos grupos señalaron que la televisión influenció en el mayor consumo de leche evaporada y frutas. Los diarios influenciaron en el consumo de alimentos de origen animal (pollo, carne, pescado) y frutas, aunque para los NPP también fueron las verduras. La radio y el internet no tuvieron influencia en el mayor consumo de algún alimento saludable. En cuanto a los alimentos no saludables, la mayoría de los adolescentes de ambos grupos indicaron que sólo la televisión influenció en el mayor consumo de golosinas (dulces, galletas, chocolates).

En términos generales, para ambos grupos de adolescentes, los programas de los medios de comunicación que más promovían el consumo de alimentos saludables fueron: Bien de Salud (canal 13), Salud en RPP (Radio Programas del Perú) y la sección Salud de los diarios El Comercio y El Trome.

Más de 70\% de los adolescentes de cada grupo indicaron que alguna vez han comprado o deseado comprar algún alimento promocionado en cualquiera de los medios de comunicación. Los productos o alimentos que compraron o que desearon comprar fueron: leche evaporada, yogurt, chocolates, galletas, dulces, chizitos, snacks y gaseosas; pero, también las bebidas industrializadas y hamburguesas, para los NPP.

El medio de comunicación que más influyó en la elección de los alimentos para ambos grupos fue la televisión, con un porcentaje mayor al 70\%. El que menos influyó para los PP fueron los diarios, con
$3,6 \%$, y para los NPP fue la radio, con 3\%. Para los adolescentes PP, la familia tiene una influencia mayor en la elección de sus alimentos $(49,7 \%)$ y para los NPP serían los medios de comunicación $(44,2 \%)$, seguido de la familia $(42,4 \%)$.

No se encontró asociación significativa entre el nivel de influencia de los medios de comunicación sobre alimentación, según la pertenencia a EPDS $(p=0,66)$; tampoco se halló diferencia en los puntajes obtenidos de la encuesta para ambos grupos $(p=0,78)$.

\section{DISCUSIÓN}

Existen estudios donde se ha demostrado que es la televisión la que más influye en la elección de los alimentos ${ }^{(13,14)}$. De igual manera, en el presente estudio, el medio de comunicación que más influyó en la elección de los alimentos para ambos grupos fue la televisión. Así, únicamente el 23,6\% y el 10,9\% de adolescentes PP y NPP, respectivamente, no reconoció alguna influencia por parte de la televisión en la elección de sus alimentos. En un estudio español se halló una cifra de 35,2\% que no reconoció alguna influencia de la televisión ${ }^{(15)}$; en dicho estudio también encontraron que 71,8\% consumía los productos que se anunciaban en la televisión, en comparación con nuestro estudio, en donde $77,6 \%$ y $72,1 \%$ de adolescentes PP y NPP, respectivamente, manifestaron haber comprado o deseado comprar algunos de los productos publicitados, que en su mayoría eran no saludables. Algo similar se encontró en un estudio australiano, donde se refiere que la publicidad de comida 'chatarra' es frecuente en la televisión; la alimentación sana, en cambio, no se promueve; muestra, además, que un mayor uso de la televisión y el ver frecuentemente comerciales se asocian independientemente con actitudes de consumo hacia la comida 'chatarra' ${ }^{(18)}$. Ante ello, surge la necesidad de cambiar el patrón publicitario de los alimentos en la televisión de nuestro país, a una donde los alimentos nutritivos sean promovidos y la comida no saludable 'chatarra' sea minimizada; así se contribuiría a reforzar la alimentación saludable y promover una vida sana, con menos riesgo de enfermedades crónicas. 




Figura 1. Niveles de influencia percibida de los medios de comunicación sobre alimentación saludable en adolescentes de nivel secundario según pertenencia al programa EPDS, distrito de Puente Piedra.

Según nuestros hallazgos, para la mitad de adolescentes, la televisión y el internet tuvieron entre una regular y mucha influencia; y para 4 de cada 10 adolescentes, la radio y los diarios tuvieron influencia semejante. Ello quiere decir que sería conveniente utilizar estos medios con mayor énfasis y con temas específicos de interés para la salud de los adolescentes y población en general, a pesar que ya se dispone en la actualidad con algunos programas y secciones periodísticas destinadas a ello. En un estudio longitudinal realizado en EE UU se determinó que, para las adolescentes, la frecuencia de las conductas saludables y sobre todo no saludables (como el ayuno, saltarse las comidas y fumar cigarrillos) y extremas (vomitar o usar laxantes) para el control de peso se incrementó con el aumento de lecturas de artículos de revistas sobre dietas y pérdida de peso ${ }^{(19)}$. Sugerimos por ello, la necesidad de intervenciones dirigidas a reducir la exposición y la importancia atribuida a los mensajes de los medios de comunicación en relación con la dieta e incorporar programas de percepción crítica para los mismos.

La mitad de los adolescentes PP consideró que la familia influenciaba más en la elección de sus alimentos, seguido de los medios de comunicación, con $28,5 \%$; los adolescentes NPP consideraron que los medios de comunicación influían en $44,2 \%$, seguido de la familia, con $42,4 \%$. Para ambos grupos, la escuela influenciaba en $10 \%$. Es evidente que la familia tiene un rol educador fundamental en ambos grupos al momento de decidir qué alimentos deben consumir en determinado momento. En un estudio realizado en adolescentes españoles de 14 a 19 años, se refiere que la información sobre alimentación la obtienen de casa $(65 \%)$, prensa, radio y TV $(19 \%)$, escuela $(17 \%)$, personal sanitario $(16 \%)$ y de amistades $(6 \%)^{(20)}$. Por el contrario, en un estudio realizado en Cuba se determinó el siguiente orden: primero la televisión, seguido de la escuela y la radio; el último lugar de influencia lo ocupó la familia ${ }^{(12)}$. Los hallazgos obtenidos en el presente estudio nos permiten inferir que los adolescentes de la población estudiada otorgan mucha importancia a la influencia de los medios de comunicación y la familia, siendo la escuela un lugar de poca influencia sobre el tema de la alimentación saludable. Esa influencia primordial de la familia a la hora de elegir alimentos puede deberse a que, aunque los hábitos de alimentación no son estables y se van modificando a lo largo de la vida, desde muy temprana edad se crea un patrón de conductas alimentarias básicas familiares ${ }^{(21,22)}$. Esta influencia familiar y la de medios de comunicación se acentúan en adolescentes y adultos, tal como lo determinó un estudio realizado en universitarios, en el que evaluaron la influencia relativa de la comunicación con los padres y la experiencia televisiva previa. Esta última siguió prediciendo las preferencias de alimentos poco saludables y la dieta en la edad adulta temprana. Además, tanto la experiencia televisiva y la crianza que recibieron de sus padres influyeron independientemente en las preferencias y la dieta ${ }^{(23)}$.

Debido a la importante influencia que ejercen los medios de comunicación y la familia, consideramos que las intervenciones educativas sobre nutrición en la televisión y la comunicación con los padres constituirían medidas efectivas para defenderse de las influencias indeseadas y para la reducción de exposición a mensajes no saludables.

Otro aspecto que cabe resaltar es que la escuela ejerce muy poca influencia al momento de elegir los alimentos para ambos grupos, a diferencia del estudio cubano ya mencionado. Si bien el programa EPDS tiene objetivos en educación de una alimentación saludable, se debe fomentar aún más la motivación, las habilidades personales en los escolares para propiciar cambios favorables, además de fortalecer acciones de promoción de la salud, tal como lo manifestaron adolescentes brasileños cuando indicaron que los materiales de promoción deberían enfatizar los beneficios inmediatos de una alimentación saludable y los mensajes de riesgo para la salud con una dieta no saludable ${ }^{(24)}$. Además, se debe tomar en cuenta las dificultades internas y externas que presenta la misma escuela para la implementación adecuada de un programa de nutrición, tal como ocurrió en un estudio en EE UU, en el cual los profesores identificaron muchas barreras ambientales para la implementación exitosa de un programa en nutrición y 
actividad física en la escuela donde laboraban, por lo que recomendaron que dichas intervenciones deberían ser coordinadas con los servicios de alimentación escolar, para que los mensajes saludables enseñados en las aulas se vieran reforzados con la disponibilidad de quioscos saludables y que estos no sean obstáculo importante para una conducta saludable en los escolares ${ }^{(25)}$.

La enseñanza en alimentación y nutrición debería partir de la conducta, problemas e intereses de los escolares en el aprendizaje significativo sobre alimentación saludable, que pueden ponerlos en práctica a través de procedimientos, utilizando estrategias didácticas que los involucren activamente así como a sus padres; de esta manera, los padres podrán inculcar a sus hijos una adecuada alimentación, ya que según el resultado de nuestro estudio, la familia tiene una influencia importante en la elección de los alimentos, aunque no solo baste con la transmisión de información sino también de motivación y demás aspectos que harán cambiar de una manera completa una conducta no saludable.

Concluimos que el nivel de influencia de los medios de comunicación sobre la alimentación saludable fue 'medio' para ambos grupos de adolescentes de nivel secundario; el promedio de puntaje fue 10,9 para los pertenecientes y 10,6 para los no pertenecientes a EPDS, sobre un máximo de 24 puntos. El medio de comunicación que más influyó en la elección de los alimentos para ambos grupos fue la televisión. Si bien la familia tiene una influencia mayor en la elección de alimentos en los pertenecientes a EPDS y los medios de comunicación en los no pertenecientes a EPDS, para ambos grupos el entorno familiar tiene un rol educador sobre el consumo de alimentos saludables.

\section{REFERENCIAS BIBLIOGRÁFICAS}

1. Ministerio de Salud, Dirección de Promoción de la Salud. Programa de Promoción de la Salud en las Instituciones Educativas. Lima: MINSA; 2005.

2. Ministerio de Salud, Dirección de Salud V Lima, Promoción de la Salud. Programa Escuelas Promotoras para el Desarrollo Sostenible de Puente Piedra, Carabayllo, Santa Rosa y Ancón, Sistematización de Experiencias. Lima: MINSA; 2008.

3. Coalición Multisectorial Perú contra el cáncer. Guías para la Promoción de la Salud orientada a la prevención y control del cáncer. Lima; 2007.

4. Ministerio de Salud, Dirección General de Promoción de la salud. Modelo de abordaje de promoción de la salud: Acciones a desarrollar en el eje temático de Alimentación y Nutrición Saludable. Lima, Documento técnico MINSA; 2006.

5. Contreras M. Refrigerios Escolares. Lima, Guía Técnica CENAN; 2006.

6. Fischer L, Espejo J. Mercadotecnia. México: Mc Graw Hill, $3^{\circ}$ Edición, 2004:360-76.

7. Brown J, Cantor J. An Agenda for research on youth and the media. J Adolesc Heatlh. 2000;275:2-7.

8. Sowden AJ, Arblaster L. Mass media interventions for preventing smoking in young people. En: The Cochrane Library, 4; Oxford: Update Software; 2001.

9. Pajuelo J. La obesidad infantil en el Perú. Anal Fac med. 2003;64(1):21-6.

10. Pajuelo J, Acevedo M. La situación nutricional de niños de 8 a 14 años en el Perú. Consensus. 2006; 11:9-16.

11. CENAN, INS, MINSA. Monitoreo Nacional de Indicadores Nutricionales. Perú; 2004.

12. Medina A, Guridi M, Ramón F, Pérez M. Comportamiento del consumo de vegetales en adolescentes de un área de salud. Revista Electrónica Psicología Científica; 2007. Disponible en: http://www.psicologiacientifica.com/bv/ psicologia-291-1-comportamiento-del-consumode-vegetales-en-adolescentes-de-u.html

13. Orozco G. El impacto educativo de la televisión no educativa. Revista Latinoamericana de estudios educativos. 1987;13(3):59-60.

14. Plasencia A. Enfoque sobre la comunicación en la gestión de proyectos comunitarios. Ciudad de la Habana: Ediciones Unión. 2003.

15. Suárez J, Navarro F. Nivel de conocimientos, actitudes y hábitos sobre alimentación y nutrición en escolares de las Palmas de Gran Canaria. Rev Esp Nutr Comunitaria 2002;8(1-2):7-18.
16. Argimón JM, Jiménez J. Métodos de investigación. Clínica y Epidemiología. 2da edición. Madrid: Ediciones Harcout. S.A; 2000:65-106.

17. Hernández R, Fernández C, Baptista P. Metodología de la Investigación. 4.a ed. Mc Graw Hill Editores. México 2006.

18. Dixon H, Scully M, Wakefield M. The effects of television advertisements for junk food versus nutritious food on children's food attitudes and preferences. Soc Sci Med. 2007;65(7):131123.

19. Van Den Berg P, Neumark D. Is dieting advice from magazines helpful or harmful? Five - years associations with weight - control behaviors and psychological outcomes in adolescents. Pediatrics. 2007;119;e30-e37.

20. Goñi C, Vilches C, Ancizu E. Factores relacionados con los comportamientos alimentarios en una población juvenil urbana. Aten Primaria. 1999;23:32-7.

21. Rivera MR. La educación en nutrición, hacia una perspectiva social en México. Rev Cubana Salud Pública. 2007;33(1):1-12.

22. Steptoe A, Wardle J. The European health and behaviour survey the development of an international study in health psychology. Psychology and Health. 1996;11:49-73.

23. Harris J, Bargh J. The relationship between television viewing and unhealthy eating: Implications for children and media interventions. Health Commun. 2009;24(7):660-73.

24. Toral N, Conti M, Slater B. Healthy eating according to teenagers: perceptions, barriers and expected characteristics of teaching materials. Cad. Saúde Pública. 2009;25(11):2386-94.

25. Bauer K, Patel A, Prokop L. Swimming upstream: faculty and staff members from urban middle schools in low-income communities describe their experience implementing nutrition and physical activity initiatives. Prev Chronic Dis. 2006;3(2):A37.

Manuscrito recibido el 15 de julio de 2010 y aceptado para publicación el 26 de setiembre de 2010.

\section{Correspondencia:}

Verónica Rosario Román Egúsquiza

Dirección: Calle Las Turquezas 1393. Urb. Angélica

Gamarra. Los Olivos. Lima, Perú.

E-mail: veroe86@hotmail.com 